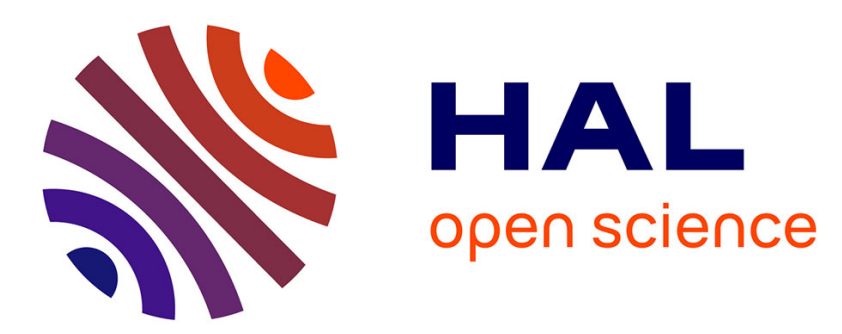

\title{
Surface quasi periodic and random structures based on nanomotor lithography for light trapping
}

Sh. Golghasemi Sorkhabi, S. Ahmadi-Kandjani, F. Cousseau, M. Loumaigne, S. Zielinska, E. Ortyl, R. Barille

\section{- To cite this version:}

Sh. Golghasemi Sorkhabi, S. Ahmadi-Kandjani, F. Cousseau, M. Loumaigne, S. Zielinska, et al.. Surface quasi periodic and random structures based on nanomotor lithography for light trapping. Journal of Applied Physics, 2017, 122 (1), pp.015303. 10.1063/1.4991480 . hal-02885280

\section{HAL Id: hal-02885280 \\ https://univ-angers.hal.science/hal-02885280}

Submitted on 30 Jun 2020

HAL is a multi-disciplinary open access archive for the deposit and dissemination of scientific research documents, whether they are published or not. The documents may come from teaching and research institutions in France or abroad, or from public or private research centers.
L'archive ouverte pluridisciplinaire HAL, est destinée au dépôt et à la diffusion de documents scientifiques de niveau recherche, publiés ou non, émanant des établissements d'enseignement et de recherche français ou étrangers, des laboratoires publics ou privés. 


\section{Surface quasi periodic and random structures based on nanomotor lithography for light trapping}

Sh. Golghasemi Sorkhabi, S. Ahmadi-Kandjani, F. Cousseau, M. Loumaigne, S. Zielinska, E. Ortyl, and R. Barille

Citation: Journal of Applied Physics 122, 015303 (2017); doi: 10.1063/1.4991480

View online: http://dx.doi.org/10.1063/1.4991480

View Table of Contents: http://aip.scitation.org/toc/jap/122/1

Published by the American Institute of Physics

\section{Articles you may be interested in}

Bistability in mushroom-type metamaterials

Journal of Applied Physics 122, 014303 (2017); 10.1063/1.4989816

Adjustment of diffraction efficiency of polarization holograms in azobenzene polymers films using electric field Journal of Applied Physics 122, 013101 (2017); 10.1063/1.4990995

Emerging photoluminescence from bilayer large-area 2D $\mathrm{MoS}_{2}$ films grown by pulsed laser deposition on different substrates

Journal of Applied Physics 122, 015304 (2017); 10.1063/1.4991490

Local structure of amorphous $\mathrm{Ag}_{5} \ln _{5} \mathrm{Sb}_{60} \mathrm{Te}_{30}$ and $\mathrm{In}_{3} \mathrm{SbTe}_{2}$ phase change materials revealed by X-ray photoelectron and Raman spectroscopic studies

Journal of Applied Physics 122, 015305 (2017); 10.1063/1.4991491

High-efficiency polarization conversion phase gradient metasurface for wideband anomalous reflection Journal of Applied Physics 122, 014501 (2017); 10.1063/1.4991505

Alignment of smectic mesogens over engineered surfaces Journal of Applied Physics 122, 014101 (2017); 10.1063/1.4989821

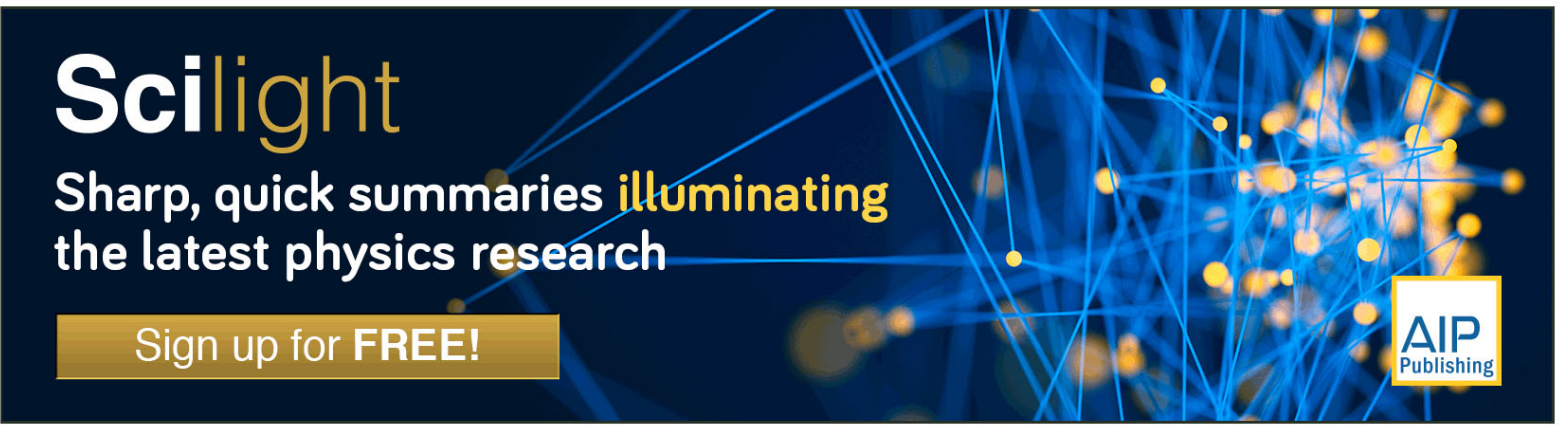




\title{
Surface quasi periodic and random structures based on nanomotor lithography for light trapping
}

\author{
Sh. Golghasemi Sorkhabi, ${ }^{1,2, a)}$ S. Ahmadi-Kandjani, ${ }^{1}$ F. Cousseau, ${ }^{2}$ M. Loumaigne, ${ }^{2}$ \\ S. Zielinska, ${ }^{3}$ E. Ortyl, ${ }^{3}$ and R. Barille ${ }^{2}$ \\ ${ }^{1}$ Research Institute for Applied Physics and Astronomy (RIAPA), University of Tabriz, 51666 Tabriz, Iran \\ ${ }^{2}$ Université d'Angers/UMR CNRS 6200, MOLTECH-Anjou, 2 bd Lavoisier, 49045 Angers, France \\ ${ }^{3}$ Department of Polymer Engineering and Technology, Wroclaw University of Technology, \\ Faculty of Chemistry, 50-370 Wroclaw, Poland
}

(Received 5 March 2017; accepted 18 June 2017; published online 6 July 2017)

\begin{abstract}
We compare the characteristics of two types of patterns obtained with two azopolymer materials: a Gaussian random pattern and a quasi-random grating pattern. The surface structurations have been obtained with a simple bottom-up technique, illuminating azopolymer thin films with a single laser beam. We demonstrate the interesting generated properties of these two surfaces. In particular, the surface with quasi-random gratings can address beam splitting for light coupling in different directions in an ultra-thin film. We use these two surfaces as a mold and replicate them on a transparent elastomeric material and demonstrate a very good light entrapment. We also show that the efficiency of light trapping is $20 \%$ better with the quasi-random gratings, than with the Gaussian random surface, and is close to 40\%. Published by AIP Publishing. [http://dx.doi.org/10.1063/1.4991480]
\end{abstract}

\section{INTRODUCTION}

Disordered photonics has experienced a burst of activities in the last two decades. Fabrication of nanostructured materials for the control of light-matter interactions has become particularly important for improving different phenomena involving a high degree of light scattering. ${ }^{1}$ For example, light emitting diodes necessitate out-coupling of light with a large directivity. ${ }^{2}$ Development of solar cells requires the reduction of the active layer. In the latter case, the need for increment of the optical absorption, in order to obtain good performance, can be managed by the use of optimized rough surfaces, to achieve a spatial and spectral broad band light trapping. Quasi-random or Gaussian random structures have been proposed as good candidates for energy harvesting.

However, it is still an inquiry if random or periodic photonic nanostructures lead to better light trapping, and how quickly they could be fabricated. ${ }^{3}$ This question has been debated and so far has not found an answer. It remains unclear if solar cells prepared on periodic or randomly textured surfaces exhibit higher efficiencies. Nevertheless, it is generally agreed that diffraction has a higher potential for enhancing the internal path length of light inside a solar cell.

Different methods with a photon control have been proposed for material and surface engineering, based on nanowires ${ }^{4}$ photonic crystal architectures ${ }^{5}$ deep reactive ion etching, chemical vapour deposition, and electron lithography. ${ }^{6}$ All these methods are costly or, depending on the required parameters, need multi-step processes. However, methods based on a surface structuration are simpler and more robust, since they are bottom-up approaches. Recently, a lithographic method based on the speckle lithography has been proposed to fabricate 2D random and quasi-random structures. The proposed method works via generation of a

a)Email: Shahla.Golghasemi@gmail.com speckle pattern and projection of this pattern onto a substrate coated with a photoresist. ${ }^{7}$ Another method uses an individual speckle diffraction phenomenon for the 1D or 2D random grating fabrication. ${ }^{8}$ The diffraction pattern of each speckle forms a micron or sub-micron size grating on the photoresist after exposure and development. In this case, even if the size of micro-gratings could be tuned, due to limitations of the speckle method, the surface is not fully covered with gratings.

Here, we propose a new method to generate a $2 \mathrm{D}$ quasi Gaussian surface and a quasi-random periodic surface (quasirandom gratings) based on molecular nano-motor lithography, where the nanoscale movements of molecules, acting as nanomotors, are fueled by the laser light. This movement can induce self-organized randomly dispersed nanopatterns on the surface of a thin film. The nanomotors are azobenzene molecules. Azobenzene derivatives are one of the most frequently studied classes of switchable compounds. The azobenzene functionality exhibits a cis-trans isomerization. The thermodynamically stable trans isomer can be converted to the cis isomer by a light stimulus, whose wavelength is in the absorption band of azobenzene, with a back isomerization induced by a thermal relaxation. Engineering azopolymer materials can lead to photoisomerization induced by light, to create selfinduced patterns on the surface of thin films. One example of such induced patterns is the production of self-organized structures leading to gratings on the surface of side-chain azobenzene grafted copolymer DR1 thin films. ${ }^{9}$ This study was the first experimental result of photoinduced spontaneous structures of azopolymers, generated with a single beam. As such, usage of azopolymers is a good choice for fabrication of random nanostructured materials for the purpose of controlled light-matter interactions, particularly for the improvement of high light scattering through different phenomena involved in the process. Through this process, different self-structured surface patterns were observed, in particular, with epoxy-based azopolymers, ${ }^{10}$ acrylic polymers bearing photoresponsive 
moieties as side chains of the polymeric backbone ${ }^{11}$ or $4 \mathrm{VP}(\mathrm{OH}-\mathrm{DMA}),{ }^{12}$ but none has ever been used for light management in photonics.

In this study, we compare two quasi random nanopatterned surfaces of azopolymer thin films, capable of being used in fabrication of devices requiring light harvesting. We discuss the advantages of both of surfaces, for energy conversion, and present two examples of applications requiring a high light coupling in a thin film. As a goal for further developments of light scattering applications, randomly textured surfaces of azopolymer materials, prepared through a self-organization process, are used as a master for replication on an elastomeric material. Manufacturing costs of this material are significantly lower, compared to random surfaces prepared via lithography or sputtering processes. Moreover, fabrication of the azopolymer master sample with different patterns can be repeated by erasing the initial surface pattern and exposing it to different light conditions in order to generate tunable patterns.

\section{MATERIALS AND METHODS}

\section{A. Azopolymer materials}

The first material is a chiral azopolymer [Fig. 1(a)], which was synthesized by polymerization of three-component mixtures of an azobenzene group containing $N$-[4-[(E)-[4-[bis(2-hydroxyethyl) amino] phenyl] azo]phenyl]sulfonyl benzamide, chiral 2,3-O-benzylidene-D-threitol, and isophorone-diisocyanate (the molar ratio of azobenzene derivative, chiraldiol, and diisocyanatein in the mixture was 1:1:2). The glass transition temperature of the azopolymer 1, determined by DSC (Electrothermal 9100), is $88^{\circ} \mathrm{C}$, and the weight of the average molecular mass of this polymer is $18400 \mathrm{~g} / \mathrm{mol}$.

The second chiral azopolymer [Fig. 1(b)] was synthesized by a radical copolymerization of an equimolar mixture of a photoisomerizable azobenzene group containing 2-[4-[(E)-[4-(acetylsulfamoyl)phenyl]azo]- $N$-methyl-anilino] ethyl 2-methylprop-2-enoate and chiral 2-methyl- $N$ -
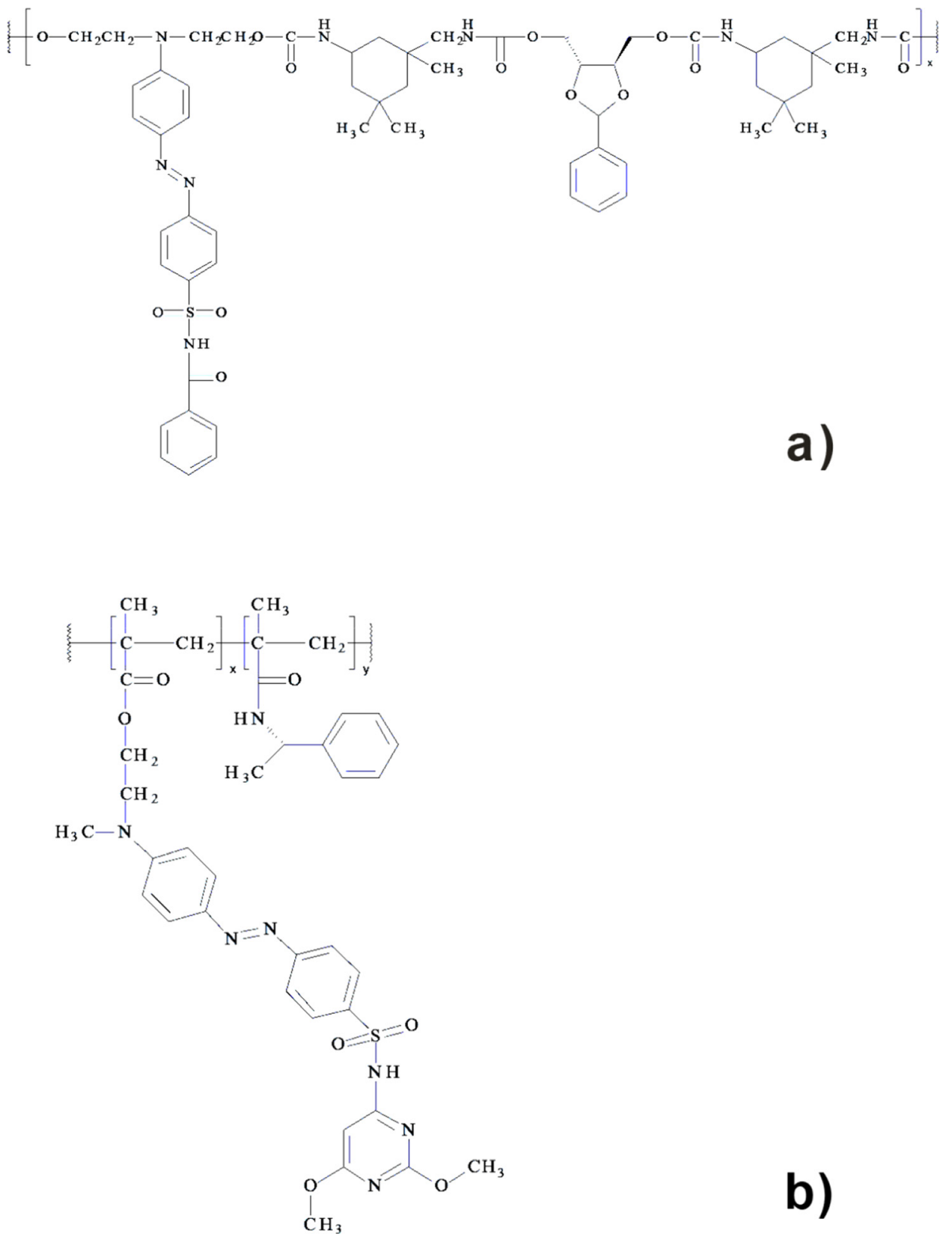

FIG. 1. (a) Chemical structure of chiral azopolymer 1 and (b) chemical structure of chiral azopolymer 2 . 
[(1S)-1-phenylethyl]prop-2-enamide. The glass transition temperature, determined by DSC, is $77^{\circ} \mathrm{C}$, and the weight of average molecular mass of this copolymer is $14700 \mathrm{~g} / \mathrm{mol}$.

The two samples have almost the same optical absorption (less than $20 \%$ change) and optical spectra (maximum absorption band at $\lambda=445 \mathrm{~nm}$ ). The samples were prepared via a spin-coating technique. The same parameters were used in the spin-coating process to fabricate the samples, and the thicknesses of the different tested samples are comparable, and are in the range of $460-510 \mathrm{~nm}$ (measured with a Dektak 32 profilometer). The chiro-optical properties of the two azopolymers were investigated by circular dichroism spectroscopy, and the results confirm the presence of chiral sub-units in the synthesized materials. Some properties of the chromophores and azopolymers were measured with quantum chemical calculations. The geometry optimization of the structures of both azopolymers was done using the Gaussian09 package [a] with a RHF method and a 3-21 g basis set. The dipole moment and polarizability values of both model structures of chromophores were similar.

\section{B. Photo-inscription of the random surface}

A diode pump solid state laser, operating at $\lambda=473 \mathrm{~nm}$, is used to excite the azopolymer close to its maximum absorption wavelength. The absorbance at the working wavelength of $473 \mathrm{~nm}$ is 0.638 . The incoming light intensity is controlled by a combination of a half wave plate and a polarizer. The sample is set perpendicular to the incident laser beam. The size of the collimated laser beam impinging on the polymer sample is adjusted with a Kepler-type afocal system and the beam is a plane wave. The sample is irradiated by a beam with a diameter size of $3 \mathrm{~mm}$ at $1 / \mathrm{e}^{2}$. The power density is $0.7 \mathrm{~W} / \mathrm{cm}^{2}$. A combination of lenses is used to measure the first order diffraction intensity, which allows the evaluation of the photoinduced structuration on the surface of the sample and the molecular rearrangement leading to a mass transport. The sample is illuminated for $15 \mathrm{~min}$, until the complete structuration of the thin film surface, which is determined by the saturation of the diffraction intensity.

\section{Random Gaussian surface simulation}

The simulated surface is characterized using terms from the probability theory such as: the height distribution function (hdf) or the statistical moments of mean and variance (or rms height $[\sigma])$. The surface variations in the lateral directions are described by the auto covariance function (acf), which describes the covariance (correlation) of the surface with translationally shifted versions. The correlation length $(\tau)$ is the typical distance between two similar features (e.g., hills or valleys). ${ }^{13}$ The simulation of the random rough surface with a Gaussian statistics is done using a method outlined in Ref. 14, where an uncorrelated distribution of surface points, using a random number generator (i.e., white noise) is convolved with a Gaussian filter to obtain the correlation. This convolution is performed more efficiently using the discrete Fast Fourier Transform (FFT) algorithm. Basically, the simulated surface can be generated by ${ }^{15}$

$$
z_{p . q}=\sum_{k=0}^{M-1} \sum_{l=0}^{N-1} h_{k \ell} \eta_{p+k, q+\ell}
$$

With $\mathrm{p}=0,1,2, \ldots,(\mathrm{M}-1), \mathrm{q}=0,1,2, \ldots,(\mathrm{N}-1) . \mathrm{h}_{\mathrm{k} \ell}$, called the FIR filter (Gaussian filter), is the coefficient defining the system, and $\eta$ is a series of Gaussian random numbers. Thus, a $\mathrm{M} \times \mathrm{N}$ point surface with a given $\mathrm{m} \times \mathrm{n}$ ACF is generated. $\mathrm{m}$ and $\mathrm{n}$ should be less than $\mathrm{M}$ and $\mathrm{N}$. We take $\mathrm{h}_{\mathrm{r}, \mathrm{s}}$ and $\mathrm{H}_{\mathrm{k}, \ell}$ as

$$
\begin{gathered}
H_{k, \ell}=\sum_{r=0}^{m-1} \sum_{s=0}^{n-1} h_{r, s} \exp \left(i 2 \pi\left[\frac{k r}{m}+\frac{l s}{n}\right]\right), \\
h_{r, s}=\frac{1}{m n} \sum_{k=0}^{m-1} \sum_{\ell=0}^{n-1} H_{k, \ell} \exp \left(-i 2 \pi\left[\frac{k r}{m}+\frac{l s}{n}\right]\right),
\end{gathered}
$$

with $\mathrm{k}_{\mathrm{r}}=0,1,2, \ldots,(\mathrm{m}-1)$ and $\ell_{\mathrm{s}}=0,1,2, \ldots,(\mathrm{n}-1)$. The FFT of Eq. (1) gives

$$
\mathrm{Z}_{\mathrm{k}, l}(\omega)=\mathrm{H}_{\mathrm{k}, l}(\omega) \mathrm{A}_{\mathrm{k}, l}(\omega),
$$

where $\mathrm{A}_{\mathrm{k}, \ell}(\omega)$ is the FFT of $\eta$ and $\mathrm{H}_{\mathrm{k}, \ell}$ is the transfer function. We take the inverse FFT of $Z_{k, \ell}(\omega)$ to obtain the simulated random surface.

\section{Extinction measurements}

The extinction measurements of the pattern replica were done with a collimated halogen lamp, covering the spectrum from $400 \mathrm{~nm}$ to $800 \mathrm{~nm}$, transmitted through the sample. The light is collected with a fiber and a collimating lens and sent to a spectrometer (Edmunds optics).

\section{E. PDMS replica of the azopolymer surface}

The polydimethylsiloxane (PDMS) prepolymer was purchased from Momentive (RTV 615). PDMS is a polymeric organosilicon compound. The PDMS prepolymer was prepared by mixing the elastomer base and the curing agent in a proper ratio $(10: 1, \mathrm{wt} / \mathrm{wt})$.

A $10 \times 10 \times 2 \mathrm{~mm}$ 3D printed frame was put on top of the thin film with the surface structure, and the prepolymer was poured into the frame and dried at room temperature for 2 days.

\section{RESULTS AND DISCUSSION}

\section{A. Quasi Gaussian surface}

The first experiment concerning surface patterns is done by illumination of the azopolymer 1 . Figure 2(a) presents the surface topography of the azopolymer 1, obtained with an atomic force microscope (AFM). The topography of the first sample is a quasi-random pattern. Illumination by a laser light induces a spontaneous self-structured surface pattern with spontaneous created cells, with diameters in the range of nanometer scale, giving a granular aspect.

The cell size has an average perimeter in the order of $2.3 \mu \mathrm{m}$. Figure 2(b) gives the Fourier transform of the surface. The lattice has a Fourier energy concentrated into a 


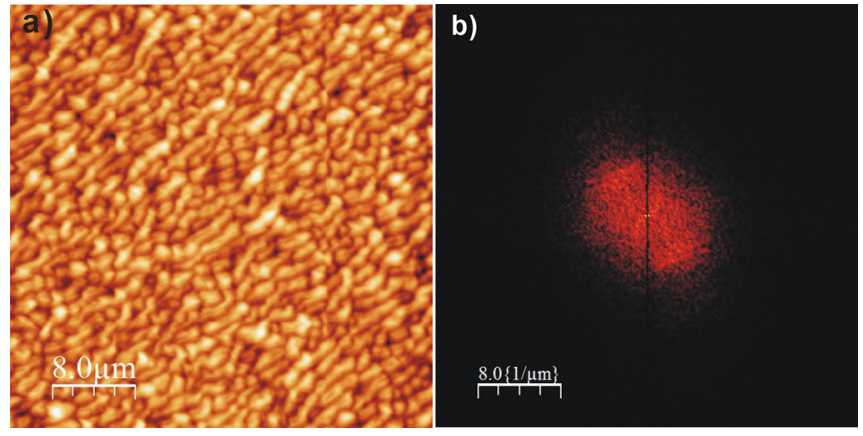

FIG. 2. (a) Surface pattern of the azopolymer 1 measured with an AFM in the contact mode and (b) Fourier transform of the surface pattern.

well-defined circular range of k-vectors with a radius value around $15.2 \mu \mathrm{m}^{-1}$.

A histogram of data, given by a statistical roughness analysis, calculated from the topographic measurements acquired by the AFM, is shown in Fig. 3(a). A fit of the roughness histogram with a Gaussian curve gives an excellent match, and the measured average height is $265 \pm 10 \mathrm{~nm}$. The granular pattern has a very small structuration anisotropy, confirmed by the symmetry of the Fourier transform. While the laser polarization was linear during the illumination, no specific correlation of the polarization in the direction of the pattern is observed.

The RMS roughness $(\sigma)$ is $82 \pm 2 \mathrm{~nm}$. In Fig. 3(b), the correlation function of surface heights is also quite close to a Gaussian curve. We measured the lateral correlation length, considering a Gaussian fit (defined as the $\mathrm{e}^{-1}$ half-width of the correlation) and we obtained a value of $\xi=510 \pm$ $10 \mathrm{~nm}$. The ratio $\sigma / \xi$ is 0.16 . With this small ratio, the slopes of the surface roughness are small. This result is confirmed by the Kurtosis parameter $(\mathrm{K})$, which is a dimensionless quantity giving a measurement of the sharpness of the height distribution function. We found $K=2.7$, close to the value 3 , for a Gaussian height distribution.

This low ratio of the surface roughness implies that if we consider a ray-tracing, following the geometrical optics, multiple scattering or shadowing effects will be attenuated and will be insignificant at small angles of light scattering (referred to the normal axis). Second order reflections can take place only for high angles of incidence.

Roughness measurements have been done on several spots on a one spin-coated sample and confirmed for different samples. The difference between all measurements is only a few percent, meaning that the spontaneous photoinduced surface patterns are reproducible, with the same conditions of solvent and spin-coating parameters.

The surface can be described, in detail, with a heigh$\mathrm{t}$-height correlation function (HHCF), $\mathrm{H}^{\circledR}$ [Fig. 3(c)]. The measured surface is explained in terms of scaling exponents. The HHCF data reach a saturation height, which can be related to the roughness of the image as well as the average grain size, by utilizing the Hurst function ${ }^{16}$ given by the following equation:

$$
H(r)=2 \sigma^{2}\left\{1-\exp \left(-\left(\frac{r}{\xi}\right)^{2 \alpha}\right)\right\}
$$

where, $\alpha$ is the Hurst parameter (or the surface roughness exponent), $\sigma$ is the surface roughness, and $\xi$ is the correlation length. The surface morphology is completely characterized by these parameters. The curve reaches a plateau and the a)

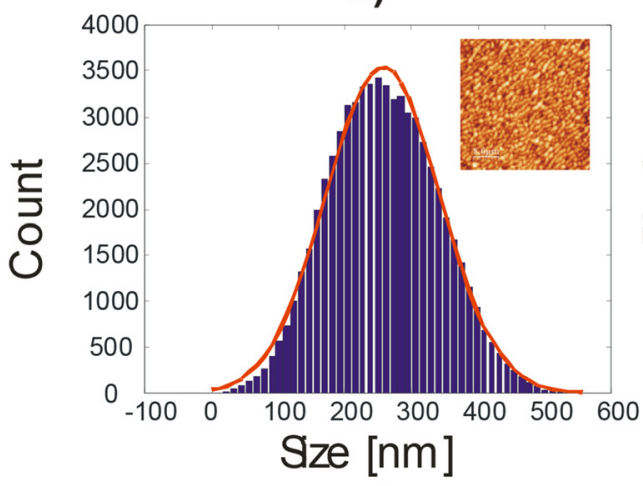

c)

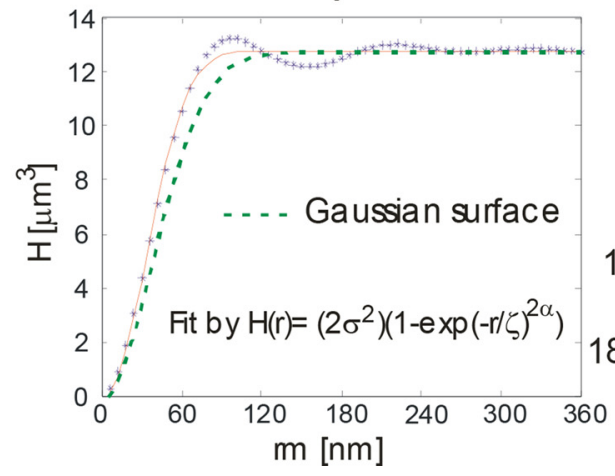

b)

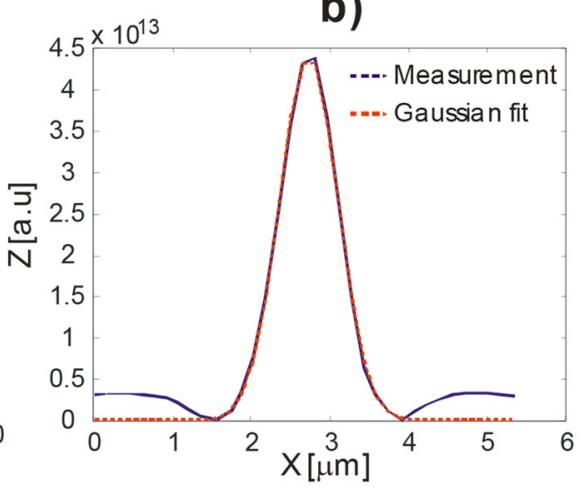

d)

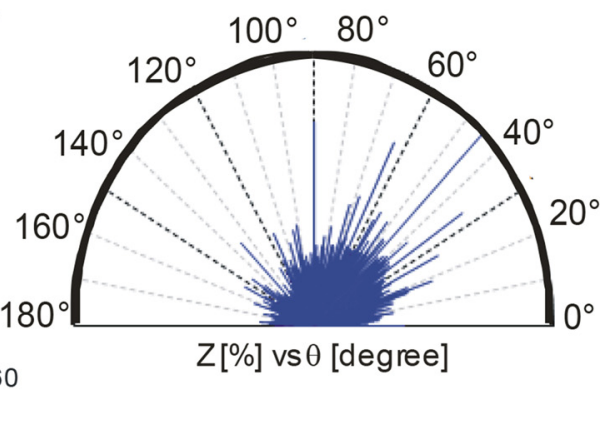

FIG. 3. (a) Histogram of the surface RMS roughness of the azopolymer 1 and its fit with a Gaussian curve, (b) auto-correlation function of the pattern surface and comparison with a pure Gaussian surface (red), (c) height-height correlation function (HHCF) and fit, and (d) directionality of the light emission from the surface. 
Hurst parameter $(\alpha)$ or surface roughness exponent influences the slope of the function before it reaches the saturation height. The surface roughness is a measure of changes in height across the surface of sample. The correlation length influences the saturation height, which is defined as the largest distance in which the height is still correlated. The surface roughness exponent, $\alpha$, allows the determination of the fractal dimension and the frequency of height fluctuations. The fractal dimension, $\mathrm{D}_{\mathrm{f}}$, corresponds to changes in the surface morphology, which occurs due to the effects of the photoinduced surface patterning. The surface roughness is linked to the fractal dimension, $D_{f}$, of the random surface by $D_{f}=D-\alpha$, where $D$ is the Euclidean dimension of the surface, $(D=3)$ that defines the geometry of the sample. We fitted the Hurst function, plotted in Fig. 3(c), to the calculated HHCF data in order to obtain the values of parameters in Eq. (5).

The fit shows a great agreement with a model of a Gaussian surface. The surface roughness exponent, $\alpha$, obtained by fitting the HHCF data of the AFM surface topography, is 0.92 . The Hurst parameter, $\alpha$, is close to 1 , meaning that the surface is smooth. The fractal dimension, $\mathrm{D}_{\mathrm{f}}$, is 2.08 .

The directionality of the emission in Fig. 3(d) gives a broadband range of angles. The emission isotropy for this random surface is $52.6 \%$. This effect is mainly due to the low value of the roughness angles and the smoothness of the surface, as it was measured with the Hurst parameter and the ratio $\sigma / \xi$. This directionality leads to an emission of light into all directions of a hemisphere, with almost a constant radiance, and consequently can be considered as quasi Lambertian.

In order to confirm that the photoinduced surface on the thin film is a random surface, obtained with a stochastic process and resulting from an acting random molecular surface process, we simulated the Gaussian surface pattern, considering the same lateral correlation length as the experimental measurements.

The result of the simulations is shown in Fig. 4. It is seen that we are unable to find information of short range or very short range periodicities in the simulated surface structure, like in the measured AFM surface [Fig. 2(a)]. By taking the fast Fourier transform (FFT) of the surface, corresponding to the image in the frequency domain, these short range

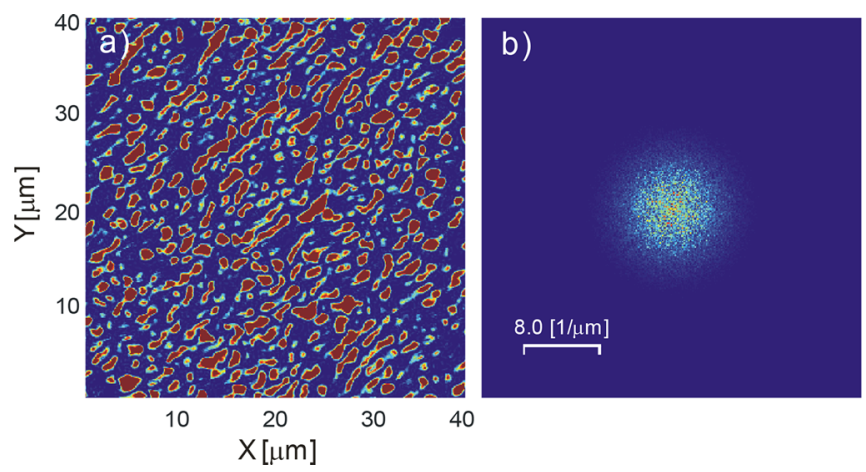

FIG. 4. (a) Simulation of a Gaussian surface considering the parameter of the photoinduced surface with the azopolymer 2 and (b) Fourier transform of the simulated surface. periodicities are particularly probed in this simulation and in the experiment. Figure 4(b) shows the FFT spectrum of the simulated random surface, which is a random distribution of data points in the frequency domain and is confined to a circular range of k-vectors, similar to the random photoinduced surface [Fig. 2(b)].

This 2D Fourier transform does not show any specific frequency distributions, characterized by two symmetrical spots generally attributed to a short range periodicity, confirming the absence of short range periodicities.

This figure is in agreement with Fig. 2(b), proving the similarity between these two surfaces. Hence, the name of quasi random surface for the self-photostructuration of the azopolymer 1 is justified.

\section{B. Quasi random grating surface}

The second experiment is done with azopolymer 2 . Figure 5(a) shows the topographical measurement of the surface after laser irradiation. The surface is spontaneously organized into sub-zones, oriented with different angles and periodic structures giving the aspect of quasi random periodic structurations. Two different types of subzones, where a periodic structure appears, are present. Each of these subzones contains a periodic structure with a pitch, defined as the distance between two maxima, of $0.51 \pm 0.2 \mu \mathrm{m}$ in the descending direction, or $0.35 \pm 0.2 \mu \mathrm{m}$ in the ascending direction. The subzones have an average length of $7.8 \pm$ $0.3 \mu \mathrm{m}$, with a maximum width of $1.6 \pm 0.2 \mu \mathrm{m}$. These zones inside the whole pattern appear to be superposed. Some parts of the sample show an overlapping between the randomly arranged sub-zones of gratings, where the periodic structures are photoinduced.

The self-organization of the thin film surface in multi gratings is the result of a mass transport due to cis-trans photoisomerization, as the light illuminates the surface. ${ }^{17}$ In our experiment, the light illumination is done by a single Gaussian laser beam, without any structuration of impinging light. The long side chain of the second azopolymer with a high $\mathrm{T}_{\mathrm{g}}$ limits the process of molecular mass transports on the surface, in order to induce a large periodic structure by increasing the local viscosity. Another possible involved phenomenon could be the free volume size in the proximity of the azobenzene chromophore leading to opto-mechanical movements. The calculated molar volume of the
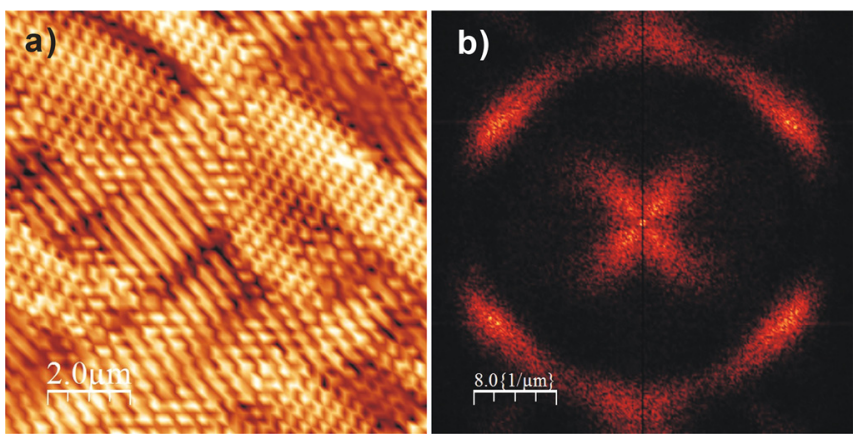

FIG. 5. (a) Surface pattern of the azopolymer 2 measured with an AFM in the contact mode and (b) Fourier transform of the surface pattern. 
sulfabenzamide azo derivative, with the Gaussian09 package, present in azopolymer 1, is $293 \mathrm{~cm}^{3} / \mathrm{mol}$. While, this value for the sulfadimethoxine azo derivative present in azopolymer 2 is distinctly lower, $263 \mathrm{~cm}^{3} / \mathrm{mol}$ (the molar volumes were calculated for trans isomers of model compounds). The lower molar volume of the photoisomerizable azobenzene derivative present in azopolymer 2 provides more free volume available for the trans-cis-trans isomerization process. This may explain the higher photoisomerization and thermal relaxation rates observed for the azopolymer 2, favouring grating patterns compared to the azopolymer 1 . Recently, we have presented results showing that, in the same condition for an azopolymer with a lower $T_{g}$, the self-organization takes place, with features having a length of $300 \pm 20 \mathrm{~nm}$, revealing the superposition of photoinduced periodic sub-structures. ${ }^{18} \mathrm{An}$ additional explanation could be based on the mechanism of phase separation of two coexistent immiscible phases in the polymer. ${ }^{19}$ The phase could be attributed to the trans and cis domains. The local concentration of trans or cis isomers serves as a morphological order parameter controlling the undulation of the surface. Adjusting this local concentration with different contributions allows simulating different spontaneous surface patterns.

The quasi random grating structuration is confirmed with the Fourier transform of the surface pattern. Figure 5(b) exhibits the Fourier spectrum of AFM measurements. The Fourier spectrum is confined to a circular range of $\mathrm{k}$ vectors, where the largest k-space distribution is due to the small structures confined in the multigratings. The low k-values in the central part of the Fourier space are due to the multigratings, layered in two different directions. We measured a maximal $\mathrm{k}$-vector value of $17.2 \mu \mathrm{m}^{-1}$, larger than the measured values obtained with quasi random nanostructures on silicon materials. ${ }^{20}$ The width of the k-vector ring is $4.5 \mu \mathrm{m}^{-1}$. Large $\mathrm{k}$-vectors are a valuable property for light trapping applications. Indeed, the exit angle, $\theta_{\mathrm{e}}$, of light diffracted by a grating surface is given by

$$
\sin \theta_{e}=q \lambda_{r} k_{m}
$$

where $\mathrm{q}$ is the diffraction order, $\lambda_{\mathrm{r}}$ is the reading wavelength, and $\mathrm{k}_{\mathrm{m}}$ is the maximal value of $\mathrm{k}$-vector in the Fourier transform of the surface pattern. If the exit angle, $\theta_{\mathrm{e}}$, is greater than $90^{\circ}$, the light cannot emerge from the surface and is trapped inside the thin film. With a value of $\mathrm{k}_{\mathrm{m}}=17.2 \mu \mathrm{m}^{-1}$, wavelengths as small as $360 \mathrm{~nm}$ do not exit the surface and are trapped inside the thin film, creating quasi-guided mode. This trapping effect is experimentally tested in Sec. IIIC.

Consequently, we can say that the surface presented in Fig. 5(a) offers an interesting trade-off between periodic and random domains: the diffraction efficiency presents simultaneously a concentration of light into very high and very low orders.

A histogram of roughness analysis data, obtained by a measurement with an AFM, is shown in Fig. 6(a). The fit of the roughness can be done with two Gaussian curves, and the histogram is in good agreement with these two curves.

The measured RMS roughness of the whole sample is $\sigma=20.6 \mathrm{~nm}$. We distinguish an average roughness height of $62.5 \mathrm{~nm}$ and $91 \mathrm{~nm}$ for the first and the second average height, respectively, given by the two roughness statistics fitted by a Gaussian curve.

The same roughness parameters were measured on different samples, fabricated in the same conditions, and the differences were in a range of few percent, confirming the a)

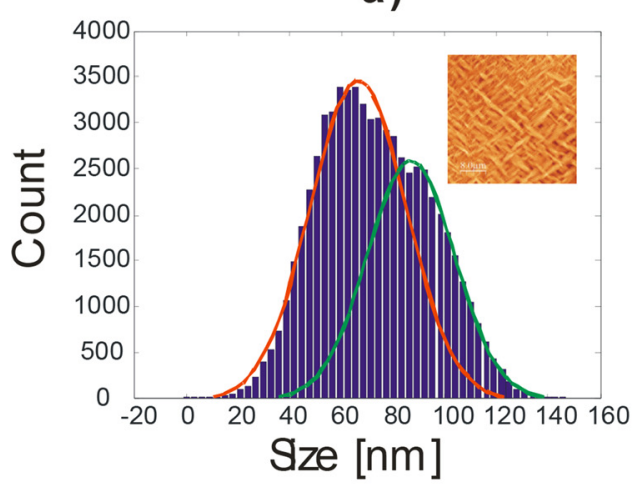

c)

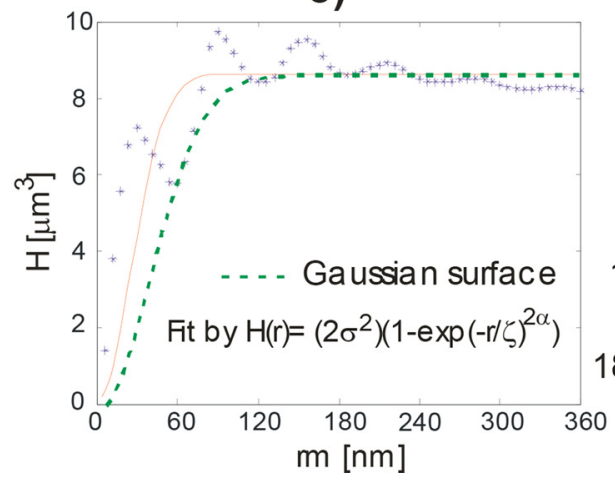

b)

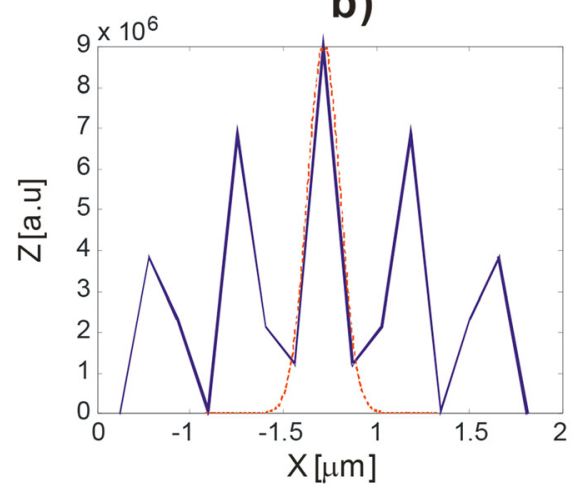

d)

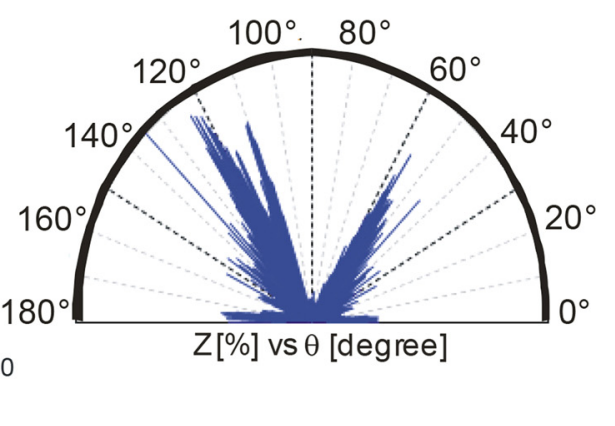

FIG. 6. (a) Histogram of the surface RMS roughness of the azopolymer 2 and its fit with a Gaussian curve, (b) auto-correlation function of the pattern surface and comparison with a pure Gaussian surface (red), (c) height-height correlation function (HHCF) and fit, and (d) directionality of the light emission from the surface. 
reproducibility of the spontaneous photoinduced patterns for this material.

The correlation function of surface heights [Fig. 6(b)] gives a lateral correlation length of $\xi=98 \pm 10 \mathrm{~nm}$. This correlation function is not Gaussian. The different peaks are explained by the periodicity of the pattern and we find the same correlation length in the other oscillations.

The ratio $\sigma / \xi$ is 0.21 . With this ratio, a large proportion of the surface becomes shadowed due to the topology for high angles of the incidence of light. ${ }^{21}$ Any valleys on the surface gets involved in multiple scattering events. So, a part of primary reflections on the surface roughness gives rise to secondary ones.

The height fluctuations occur at different length scales. The Hurst parameter corresponds to the frequency of height fluctuations. The surface roughness exponent parameter obtained from the fitting of the AFM scan is $\alpha=0.63$, far from the value $\alpha=1$, indicating a rough surface. Figure $6(\mathrm{~d})$ shows the light directionality of the quasi-random gratings on the surface. We calculated an isotropy of $18.3 \%$ in the emission of light from the surface. The directionality of the emission covers mostly four directions of the space. This anisotropic emission is due to the large random variations of micrometer length gratings on the surface, associated with a nano-patterning with a honeycomb form inside the gratings. However, close to the surface, the directionality of the emission is almost in the surface plane, allowing the reduction of the thickness of the trapping film. The surface texture model can be thought as the superposition of emission from gratings formed by slits, separated from one another by different distances. $^{22}$

\section{Quasi guided modes into the thin film}

In order to test the validity of light coupling through quasi-guided modes into the thin film, we have created four additional grating structures next to the quasi-random grating surface [Fig. 7(a)]. First, the central pattern corresponding to quasi random gratings is spontaneously created by illuminating the azopolymer 2 with a single beam. Afterwards, four gratings are made with a laser interference pattern, illuminating the sample through a pinhole. The sample was rotated in order to inscribe the 4 gratings, one by one, at the cardinal

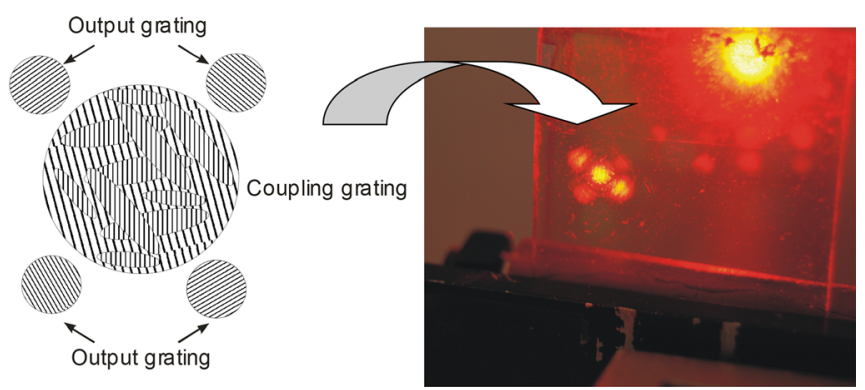

FIG. 7. Four gratings were inscribed in the cardinal direction in order to show the mode coupling of the light in all the directions and the outcoupling light by the 4 gratings. The picture on the right shows that light (He-Ne laser) injected on the quasi random grating surface (coupling grating) is outcoupled by the output gratings. This confirms the existence of quasi guided modes in the thin film. points, close to the central quasi random grating surface. The laser interference pattern is obtained by separating the beam from a linearly polarized laser beam via a beam splitter and recombining them with two mirrors.

The positions of these four gratings match the positions of the diffracted spots given by the Fourier transform of the surface pattern [Fig. 5(b)]. The pitch of these four gratings, measured from AFM images, is $\Lambda=0.9 \pm 0.05 \mu \mathrm{m}$ with an amplitude of $100 \pm 10 \mathrm{~nm}$. After inscription of these gratings, a He-Ne laser is used to illuminate the central quasirandom grating pattern of the surface. We used a He-Ne laser in order to avoid possible photoinduced changes applied on the surface by the laser.

As it can be seen in Fig. 7(b), the central pattern diffracts the incoming light with such big angles that a part of the incoming light cannot directly exit the film. However, the trapped light can excite the quasi-guided modes into the thin film. The guided energy is then outcoupled by the 4 satellite gratings. The variation of light intensity in the four gratings is due to amplitude inhomogeneities in the central pattern, affecting the total repartition of the extracted light into the four gratings. Tilting the sample, with respect to the incident angle, is important and can also affect the extracted light. The intensity of the light coming from the gratings can be controlled by changing the incident angle. In addition to applications for light harvesting, the quasi random-grating surface can also be seen as a four beam splitter for thin films.

\section{Light trapping efficiency measurements}

Due to the surface relief features, the surface-patterned azopolymer films can be used directly as a soft lithographic master to cast molds. We replicated the photoinduced surface of the chiral azopolymers 1 and 2 on a widely used elastomeric material [poly(dimethylsiloxane (PDMS)]. This material has a low interfacial free energy. The PDMS sample is largely transparent, since it has an optical absorption of $0.04 \%$ and a transmission above $95 \%$ in the visible region of the spectrum. The printed replicas showed the same random structures as the masters, along with the same relief depths. Figure 8 shows the photographic images of the stamps. Brilliant and iridescent colors, resulting from the diffraction of the surface gratings, can be observed for the replica of the quasi-random grating surface (sample A in Fig. 8).

Taking the plane PDMS surface as a reference, we measured the light extinction, induced by the patterned surface, for both materials. We found an increase of the light extinction ratio of $28 \%$ and $41 \%$ for the quasi Gaussian surface and the quasi-random grating surface, respectively. This light extinction is homogeneous over the visible spectrum, which is a valuable property for light harvesting. Since no absorption can occur inside the PDMS material, this extinction is due to light scattered by the random surface (for both surfaces) and light trapped into the thin film (only for the quasi random grating surface).

Consequently, light extinction here has to been understood in the more general concept of light harvesting. Therefore, from the measurements, we can say that the quasi-random grating surface is $20 \%$ more efficient than the 

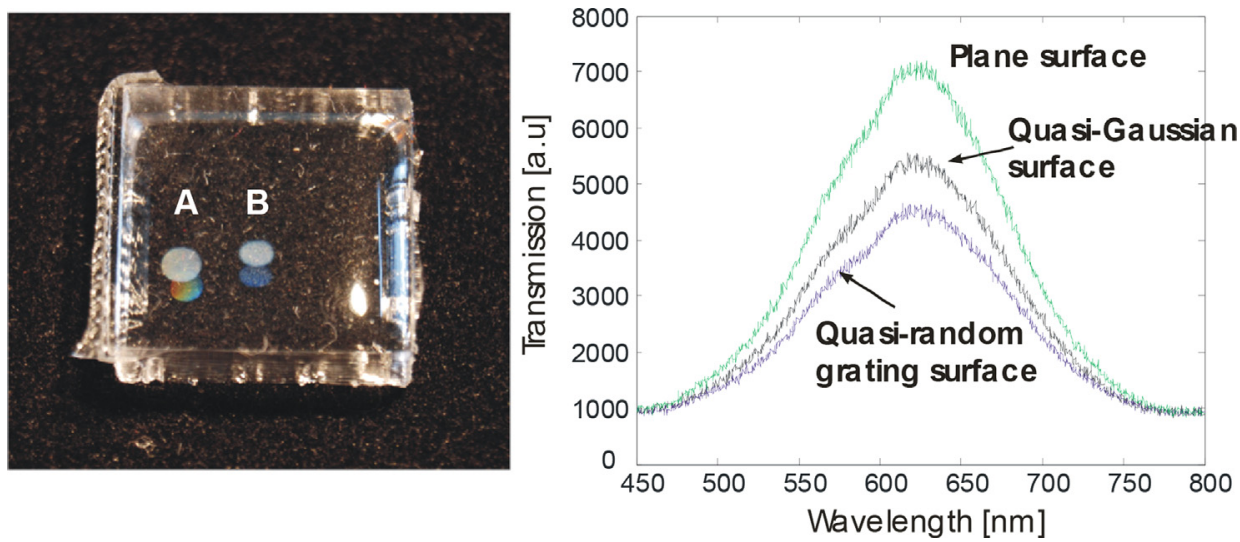

FIG. 8. (a) Replica modeling of the surface pattern of the azopolymer 1 and azopolymer 2 on an elastomeric material $\mathrm{A}$ and $\mathrm{B}$, respectively (A is the quasi-random grating surface). (b) Transmission spectrum of the elastomeric surface with quasi-random gratings, a Gaussian surface, and comparison with the reference plane surface.

quasi Gaussian surface for light harvesting. This effect is due to the random gratings associated with honey-comb structures inside the small gratings. These are similar or quasi better than the results obtained with a photonics crystal. ${ }^{20,23}$ Let us stress out again that in the quasi random grating structure, we combine the role of a random surface with the one of multigratings to observe a large concentration of energy in higher orders, approaching the Lambertian limit. This replicated patterned surface can generate a good light trap.

\section{CONCLUSION}

In conclusion, we have presented a simple and cost effective way to prepare a quasi Gaussian surface and a quasi random grating surface using a self-photoinduced process on two chiral azopolymer thin films. The creation of a more interesting quasi random grating surface is possible, via the combination of a photoisomerization process associated with a good choice of the azopolymer material, in particular, with the correct choice of the copolymer function and the grafted azobenzene. The quasi random grating surface has the same advantages as the quasi Gaussian surface for light scattering but, as it was experimentally shown, additionally, it allows an induction of an isotropic mode coupling of light into the thin film, increasing its efficiency by $20 \%$ for light harvesting.

We have highlighted the applicability of the quasi random grating surface for realistic devices. The replication of the surface pattern on a PDMS surface (a transparent and cost effective material) shows how easily this technique can be used to modify samples like Organic light-emitting diode (OLEDs) or solar cells for a better efficiency of light diffusion or light harvesting. ${ }^{24-27}$ Furthermore, we note that the primary photoinduced surface can be erased for re-illumination and regeneration of a new photostructured surface. Finally, the chemical structure of the azobenzene grafted copolymer can be engineered, leading to new observational patterns.

\section{ACKNOWLEDGMENTS}

We thank Romain Mallet (SCIAM - University of Angers) for the use of their equipment.

Calculations have been carried out using resources provided by the Wroclaw Centre for Networking and Supercomputing (http://wcss.pl), Grant No. 271.
${ }^{1}$ R. Dewan, S. Shrestha, V. Jovanov, J. Hüpkes, K. Bittkau, and D. Knipp, "Random versus periodic: Determining light trapping of randomly textured thin film solar cells by the super position of periodic surface textures," Sol. Energy Mater. Sol. Cells 143, 183-189 (2015).

${ }^{2}$ Y. Park, F. Nehm, L. Müller-Meskamp, K. Vandewal, and K. Leo, "Optical display film as flexible and light trapping substrate for organic photovoltaics," Opt. Express 24(10), A974-A980 (2016).

${ }^{3}$ C. Battaglia, C.-M. Hsu, K. Soderstrom, J. Escarre, F.-J. Haug, M. Charriere, M. Boccard, M. Despeisse, D. T. L. Alexander, M. Cantoni, Y. Cui, and C. Ballif, "Light trapping in solar cells: Can periodic beat random," ACS Nano 6(3), 2790-2797 (2012).

${ }^{4}$ Z.-Q. Fang, H.-L. Zhu, Y.-Y. Li, Z. L. J.-Q. Dai, C. Preston, S. Garner, P. Cimo, X.-S. Chai, G. Chen, and L.-B. Hu, "Light management in flexible glass by wood cellulose coating," Sci. Rep. 4, 5842 (2014).

${ }^{5}$ A. Oskooi, Y. Tanaka, and S. Noda, "Tandem photonic-crystal thin films surpassing Lambertian light-trapping limit over broad bandwidth and angular range," Appl. Phys. Lett. 104, 091121 (2014).

${ }^{6}$ M. C. Gather and S. Reineke, "Recent advances in light outcoupling from white organic light-emitting diodes," J. Photonics Energy 5, 057607 (2015).

${ }^{7}$ J. Bingi and V. M. Murukeshan, "Speckle lithography for fabricating Gaussian, quasi-random 2D structures and black silicon structures," Sci. Rep. 5, 18452 (2015).

${ }^{8}$ J. Bingi and V. M. Murukeshan, "Individual speckle diffraction based 1D and 2D random grating fabrication for detector and solar energy harvesting applications," Sci. Rep 6, 20501 (2016).

${ }^{9}$ C. Hubert, C. Fiorini-Debuisschert, L. Rocha, P. Raimond, and J.-M. Nunzi, "Spontaneous photoinduced patterning of azo-dye polymer films: The facts," JOSA B 24(8), 1839-1846 (2007).

${ }^{10} \mathrm{X}$. L. Wang, J. J. Yin, and X. G. Wang, "Self-structured surface patterns on epoxy-based azo polymer films induced by laser light irradiation," Macromolecules 44, 6856-6867 (2011).

${ }^{11}$ A. Ambrosio, S. Girardo, A. Camposeo, D. Pisignano, and P. Maddalena, "Controlling spontaneous surface structuring of azobenzene-containing polymers for large-scale nano-lithography of functional substrates," Appl. Phys. Lett. 102, 093102 (2013).

${ }^{12}$ A. Sobolewska and S. Bartkiewicz, "Single beam test (SBT) as a criterion for the resolution of holographic recording," J. Mater. Chem. C 3, 5616-5620 (2015).

${ }^{13}$ D. Bergstrom, J. Powel, and A. F. H. Kaplan, "A ray-tracing analysis of the absorption of light by smooth and rough metal surfaces," J. Appl. Phys. 101, 113504 (2007).

${ }^{14}$ Y. Z. Hu and K. tonder, "Simulation of 3-D random rough surface by 2-D digital filter and Fourier analysis," Int. J. Mach. Tools Manuf. 32(12), 83-90 (1992).

${ }^{15}$ J.-J. Wu, "Simulation of rough surfaces with FFT," Tribol. Int. 33, 47-58 (2000).

${ }^{16}$ N. Rahmani and R. S. Dariani, "Porosity-dependent fractal nature of the porous silicon surface," AIP Adv. 5, 077112 (2015).

${ }^{17}$ L. Mazaheri, R. G. Sabat, O. Lebel, and J.-M. Nunzi, "Unraveling the nucleation and growth of spontaneous surface relief gratings," Opt. Mater. 62, 378-391 (2016)

${ }^{18}$ V. Teboul, R. Barillé, P. Tajalli, S. Ahmadi-Kandjani, H. Tajalli, S. Zielinska, and E. Ortyl, "Light mediated emergence of surface patterns in azopolymers at low temperature," Soft Matter 11, 6444-6449 (2015).

${ }^{19}$ H. Galinski, A. Ambrosioa, P. Maddalenac, I. Schenkerd, R. Spolenakb, and F. Capassoa, "Instability-induced pattern formation of photoactivated functional polymers," PNAS 111, 17017-17022 (2014). 
${ }^{20}$ E. R. Martins, J. Li, Y. Liu, V. Depauw, Z. Chen, J. Zhou, and T. E. Krauss, "Deterministic quasi-random nanotructures for photon control," Nat. Commun. 4, 2665 (2013).

${ }^{21}$ K. A. O’Donnell and E. R. Mendez, "Experimental study of scattering from characterized random surfaces," J. Opt. Soc. Am. A 4(7), 1194-1205 (1987).

${ }^{22}$ I. Simonsen, "Optics of surface disordered systems," Eur. Phys. J. Spec. Top. 181, 1-103 (2010).

${ }^{23}$ V. Gianapani, O. D. Miller, and E. Yablonovitch, "Light trapping textures designed by electromagnetic optimization for subwavelength thick solar cells," IEEE J. Photovoltaics 4(1), 175-182 (2014).
${ }^{24}$ P. Kowalczewski, M. Liscidini, and L. C. Andreani, "Engineering Gaussian disorder at rough interfaces for light trapping in thin-film solar cells," Opt. Lett. 37, 4868-4870 (2012).

${ }^{25}$ I. Schnitzer and E. Tablonovitch, "30\% external quantum efficiency from surface textured, thin-film light-emitting diodes," Appl. Phys. Lett. 63(16), 2174-2176 (1993).

${ }^{26} \mathrm{P}$. Beckman and A. Spizzichino, The Scattering of Electromagnetic Waves from Rough Surfaces (Pergamon press, 1963).

${ }^{27}$ C. Hubert, C. Fiorini-Debuisschert, I. Hassiaoui, L. Rocha, P. Raimond, and J. M. Nunzi, Appl. Phys. Lett. 87, 191105 (2005). 